Europhys. Lett., 45 (4), pp. 418-423 (1999)

\title{
Microscopic theory of the Aharonov-Bohm effect
}

\author{
E. Santos ${ }^{1}(*)$ and I. $\operatorname{GonZalO}^{2}\left(^{* *}\right)$ \\ ${ }^{1}$ Departamento de Física Moderna, Universidad de Cantabria - 39005-Santander, Spain \\ ${ }^{2}$ Departamento de Optica, Facultad de Ciencias Físicas, Universidad Complutense \\ 28040-Madrid, Spain
}

(received 8 July 1998; accepted 8 December 1998)

PACS. 03.65Bz - Foundations, theory of measurement, miscellaneous theories (including Aharonov-Bohm effect, Bell inequalities, Berry's phase).

PACS. 12.20-m- Quantum electrodynamics.

\begin{abstract}
A fully quantum theory of the Aharonov-Bohm effect is presented. It is based on the interaction, mediated by virtual photon exchange, between the traveling electron and the atomic magnetic dipoles of an infinitely long permanent magnet. The calculation involves second-order time-dependent perturbation theory. As expected, the relative phase between the two states - corresponding to the two alternative paths - interfering on the screen agrees with that predicted in the usual theory. However our method may allow to get corrections, for instance due to the size and shape of the electron wave packet, the possible soft-photon emission or the excitation of the atoms in the magnet.
\end{abstract}

Introduction. - The Aharonov-Bohm (AB) effect is, essentially, the change in phase of the wave function of an electron passing near a solenoid or magnet, through a region where there is no electric or magnetic field but there is a nonzero vector potential (for a review see Peshkin and Tonomura [1]). The relative phase between the two states of the charged particle interfering on the screen (see fig. 1) produces changes in the quantity $\Delta \varphi=(q / \hbar) \Phi$, where $q$ is the charge of the particle and $\Phi$ is the magnetic flux in SI units (webers).

The standard theory of the $\mathrm{AB}$ effect involves a semiclassical approximation. In fact, the solenoid or magnet is treated classically. It produces a classical magnetic field inside the solenoid and a classical vector potential. Only the electron is treated as a quantum particle. The procedure is plausibly quite accurate because the solenoid is certainly macroscopic but, from a fundamental point of view, it is rather inconvenient because it gives rise to a paradoxical situation. On the one hand it is claimed that the potential is more fundamental than the field in quantum mechanics, in sharp contrast with classical physics. But, on the other hand, the potential is treated classically, not as an operator function in a Hilbert space.

The problem is aggravated by the long controversy that has taken place about the correct interpretation of the $\mathrm{AB}$ effect. It has lasted more than 30 years since the discovery of the effect $[2,3]$ and involved hundreds of papers in scientific journals. Some authors have claimed that the AB effect simply does not exist [4]. Other authors have assumed that there is some force on the electron, in addition to the Lorentz force $[5,6]$. Although the AB affect has been

$\left.{ }^{*}\right)$ E-mail: santose@besaya.unican.es

$\left({ }^{* *}\right)$ E-mail: igonzalo@eucmax.sim.ucm.es

(C) EDP Sciences 


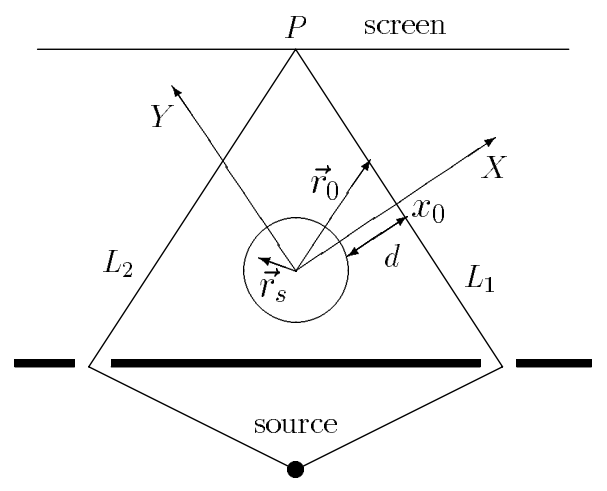

Fig. 1. - The two possible paths, $L_{1}, L_{2}$, for an electron arriving at $P$.

confirmed experimentally [7,1], there are people claiming that what is experimentally found is due to some small magnetic fields unavoidably present outside the solenoid due to its finite length or to some imperfections.

In the present article we shall see that in a consistent quantum calculation the controversy about what is relevant, the field or the potential, loses sense because they both are operators which may be written as linear combinations of creation and destruction operators of photons. Also all interactions are local as is always the case in quantum electrodynamics.

Our aim is to calculate the change in phase of an electron passing near, but outside, an infinitely long cylinder with a constant magnetic field inside pointing in the direction of the cylinder axis. For the purpose of making a fully quantum treatment it is more simple to have a model with a magnet rather than a solenoid. Consequently, we consider a homogeneous infinitely long cylindrical permanent magnet consisting of identical atomic magnetic-dipole moments, all of them oriented along the axis of the magnet. We treat the atomic dipoles as quantum objects, and the interaction between them and the electron takes place via the emission, and subsequent absorption, of virtual photons. In the formalism this leads to a calculation involving second-order perturbation theory. An additional advantage of our treatment is that causality is never violated, something that has not always been clear in the study of the AB effect.

Theory. - Let us take the $Z$-axis along the cylindrical axis of the magnet. For the sake of simplicity we assume that the magnet is composed by $N$ identical atoms or molecules per unit volume, all in the same eigenstate of angular momentum $J_{z}$ with eigenvalue $\hbar M$, so that each one of them has a magnetic-dipole moment in the $Z$-direction given by $g \mu_{\mathrm{B}} M$, where $\mu_{\mathrm{B}}$ is Bohr's magneton, and $g$ is the gyromagnetic ratio.

In a plane perpendicular to the $Z$-axis, a beam of coherent electrons is sent to a screen. An electron arrives at one point, for example $P$ (see fig. 1), but there are two possible paths, one at each side of the magnet, and interference may be produced. The origin of coordinates is taken in this plane, in the center of the circular section of the magnet.

We deal first with the electron following the path $L_{1}$. We take the $Y$-axis parallel to the direction of this path as shown in fig. 1.

The total Hamiltonian of the system is

$$
H=H_{0}+H_{\mathrm{int}}, \quad H_{0} \equiv H_{0}^{(\mathrm{e})}+H_{0}^{(\mathrm{m})}+H_{\mathrm{rad}}, \quad H_{\mathrm{int}} \equiv H_{\mathrm{int}}^{(\mathrm{e})}+H_{\mathrm{int}}^{(\mathrm{m})},
$$

where $H_{0}^{(\mathrm{e})}=\vec{p}^{2} /(2 m)$ is the Hamiltonian of the free electron, $H_{0}^{(\mathrm{m})}$ that of the atoms in the 
magnet, $H_{\mathrm{rad}}$ is the Hamiltonian of the free radiation field, and $H_{\mathrm{int}}^{(\mathrm{e})}, H_{\mathrm{int}}^{(\mathrm{m})}$ account for the interaction of the radiation field with the electron and with the magnet, respectively:

$$
H_{\mathrm{int}}^{(\mathrm{e})} \equiv \frac{e}{m} \vec{p} \cdot \vec{A}\left(\vec{r}_{\mathrm{e}}\right),(e=|e|), \quad H_{\mathrm{int}}^{(\mathrm{m})} \equiv \sum_{s} g \frac{\mu_{\mathrm{B}}}{\hbar} J_{z}^{(s)} \vec{u}_{z} \cdot \vec{B}\left(\vec{r}_{s}\right) .
$$

As is usual in the study of the AB effect, we ignore the electron spin. We consider that the traveling electron is not prepared in a specific spin state so that $\left\langle\vec{s}_{\mathrm{e}}\right\rangle=0$ and the value of the interaction of the electron with the magnetic field $\vec{B}$ of the radiation is zero. In eq. (2), $\vec{p}$ is the momentum of the electron and $\vec{A}$ the vector potential of the radiation. Concerning the magnet, we have taken into account that the electric-dipole transitions in the atoms give the same contribution - and with the same sign - either if the traveling electron follows the path $L_{1}$ or if it follows the path $L_{2}$. Consequently, the Hamiltonian term corresponding to these transitions is omitted in $H_{\mathrm{int}}^{(\mathrm{m})}$. However, the magnetic-dipole transitions induced by $\vec{B}$ in the atomic dipoles, numbered by $s$, are relevant due to their different contribution - opposite signwhen the electron follows the path $L_{1}$ or $L_{2}$. The vector potential expressed as a plane-wave expansion and in SI units is given by $\vec{A}(\vec{r})=\sum_{\vec{k}, \vec{\epsilon}}\left(\hbar / 2 \omega \epsilon_{0} V\right)^{1 / 2} \vec{\epsilon}\left(e^{i \vec{k} \cdot \vec{r}} a_{\vec{k}, \vec{\epsilon}}+e^{-i \vec{k} \cdot \vec{r}} a_{\vec{k}, \vec{\epsilon}}^{+}\right)$, where $\vec{k}$ and $\vec{\epsilon}$ are the wave vector and the polarization of the photon, respectively. Then $\vec{B}(\vec{r})=\vec{\nabla} \times \vec{A}(\vec{r})$.

The state at the initial time is $|\Psi(-T)\rangle \equiv\left|\psi_{\mathrm{e}}(-T)\right\rangle|\chi(-T)\rangle|0\rangle$, where $\left|\psi_{\mathrm{e}}\right\rangle$ is the wave packet of the free traveling electron, $|\chi\rangle$ is the state of the permanent magnet, and $|0\rangle$ is the vacuum radiation state (of zero photons). We consider the electron far from the magnet in the instant $t=-T$, passing close to the magnet, at the point $\left(x_{0}, 0,0\right)$, at the time $t=0$, and arriving to the screen at time $t=T$. Eventually we shall take $T \rightarrow \infty$. We may ignore cases in which a real photon is created because they have low probability. Therefore the final state will be $|\Psi(T)\rangle \equiv\left|\psi_{\mathrm{e}}(T)\right\rangle|\chi(T)\rangle|0\rangle$. As our aim is to calculate the change of phase of the electron wave function due to the interaction, it is convenient to work in the interaction picture, where the unperturbed state vectors do not change. Our calculation will show that the magnet remains in the ground state with great probability. Therefore the scalar product of the initial state vector times the final one gives the change of the electron wave function, due to the interaction with the magnet. We may write

$$
\langle\Psi(-T) \mid \Psi(T)\rangle=e^{i \varphi_{L_{1}}}
$$

which we shall see has unit modulus, i.e., $\varphi_{L_{1}}$ is real and we may identify it with the change in phase of the electron wave function.

Using standard time-dependent perturbation theory we get, to second order,

$$
|\Psi(T)\rangle=\left[1+\left(\frac{i}{\hbar}\right)^{2} \int_{-T}^{T} \mathrm{~d} t \int_{-T}^{t} \mathrm{~d} t^{\prime} H_{\mathrm{I}}\left(t^{\prime}\right) H_{\mathrm{I}}(t)\right]|\Psi(-T)\rangle
$$

where $H_{\mathrm{I}}(t) \equiv e^{i H_{0}(t+T) / \hbar} H_{\mathrm{int}} e^{-i H_{0}(t+T) / \hbar}$. It is easy to see that there is no first-order contribution if the final radiation state is the vacuum. If we put (4) in (3), we get

$$
e^{i \varphi_{L_{1}}}-1 \approx i \varphi_{L_{1}}=\left(\frac{i}{\hbar}\right)^{2} \int_{-T}^{T} \mathrm{~d} t \int_{-T}^{t} \mathrm{~d} t^{\prime}\left\langle\Psi_{\mathrm{S}}(t)\left|e^{i H_{0}\left(t-t^{\prime}\right) / \hbar} H_{\mathrm{int}} e^{i H_{0}\left(t^{\prime}-t\right) / \hbar} H_{\mathrm{int}}\right| \Psi_{\mathrm{S}}(t)\right\rangle,
$$

where we have defined ( $\mathrm{S}$ is for Schrödinger picture) $\left|\Psi_{\mathrm{S}}(t)\right\rangle=e^{-i H_{0}(t+T) / \hbar}\left|\Psi_{\mathrm{S}}(-T)\right\rangle$, the state $\left|\Psi_{\mathrm{S}}(-T)\right\rangle$ being the same in the Schrödinger and interaction pictures. We now introduce in (5) the sums $\sum_{i}|i\rangle\left\langle i\left|, \sum_{f}\right| f\right\rangle\left\langle f\left|, \sum_{n}\right| n\right\rangle\langle n|$, with $|i\rangle \equiv\left|\vec{p}_{i}\right\rangle|\chi\rangle|0\rangle,|f\rangle \equiv\left|\vec{p}_{f}\right\rangle|\chi\rangle|0\rangle,|n\rangle \equiv$ 
$\left|\vec{p}_{n}\right\rangle|\chi\rangle|\vec{k}, \vec{\epsilon}\rangle$, where $\left|\vec{p}_{i}\right\rangle,\left|\vec{p}_{f}\right\rangle,\left|\vec{p}_{n}\right\rangle$ are eigenstates of the electron momentum, and we recall that $|0\rangle$ is the zero photon state. Then, we have

$$
\varphi_{L_{1}}=\frac{i}{\hbar^{2}} \int_{-T}^{+T} \mathrm{~d} t \sum_{f, n, i}\left\langle\Psi_{\mathrm{S}}(t) \mid f\right\rangle\left\langle f\left|H_{\mathrm{int}}\right| n\right\rangle\left\langle n\left|H_{\mathrm{int}}\right| i\right\rangle\left\langle i \mid \Psi_{\mathrm{S}}(t)\right\rangle \int_{t}^{T} \mathrm{~d} t^{\prime} e^{i\left(E_{n}-E_{f}\right)\left(t^{\prime}-t\right) / \hbar}
$$

where $E_{n}=E_{\chi}+E_{\vec{p}_{n}}+\hbar \omega$, with $\omega=c k$, and $E_{f}=E_{\chi}+E_{\vec{p}_{f}}$. We shall consider $T \rightarrow \infty$ and use the standard prescription $E_{n}-E_{f} \rightarrow E_{n}-E_{f}+i \epsilon$ with $\epsilon>0$, which allows us to neglect the upper limit of the last integral of (6), obtaining

$$
\varphi_{L_{1}}=-\frac{1}{\hbar} \int_{-\infty}^{+\infty} \mathrm{d} t \sum_{f, n, i} \frac{\left\langle\Psi_{\mathrm{S}}(t) \mid f\right\rangle\left\langle f\left|H_{\mathrm{int}}\right| n\right\rangle\left\langle n\left|H_{\mathrm{int}}\right| i\right\rangle\left\langle i \mid \Psi_{\mathrm{S}}(t)\right\rangle}{E_{n}-E_{f}} .
$$

It can be seen that

$$
\begin{aligned}
\sum_{n}\left\langle f\left|H_{\mathrm{int}}\right| n\right\rangle\left\langle n\left|H_{\mathrm{int}}\right| i\right\rangle= & \left\langle 0 ; \vec{p}_{f} ; \chi\left|H_{\mathrm{int}}^{(\mathrm{m})}\right| \chi ; \vec{p}_{f} ; \vec{k}, \vec{\epsilon}\right\rangle\left\langle\vec{k}, \vec{\epsilon} ; \vec{p}_{f} ; \chi\left|H_{\mathrm{int}}^{(\mathrm{e})}\right| \chi ; \vec{p}_{i} ; 0\right\rangle+ \\
& +\left\langle 0 ; \vec{p}_{f} ; \chi\left|H_{\mathrm{int}}^{(\mathrm{e})}\right| \chi ; \vec{p}_{i} ; \vec{k}, \vec{\epsilon}\right\rangle\left\langle\vec{k}, \vec{\epsilon} ; \vec{p}_{i} ; \chi\left|H_{\mathrm{int}}^{(\mathrm{m})}\right| \chi ; \vec{p}_{i} ; 0\right\rangle .
\end{aligned}
$$

These two terms are the only ones contributing to the electron-magnet interaction. Note that the direct terms $\left\langle f\left|H_{\mathrm{int}}^{(\mathrm{m})}\right| n\right\rangle\left\langle n\left|H_{\mathrm{int}}^{(\mathrm{m})}\right| i\right\rangle$ and $\left\langle f\left|H_{\mathrm{int}}^{(\mathrm{e})}\right| n\right\rangle\left\langle n\left|H_{\mathrm{int}}^{(\mathrm{e})}\right| i\right\rangle$, when $\vec{p}_{n}=\vec{p}_{i}=\vec{p}_{f}$, contribute to the respective self-energies of the magnet and of the electron, and can thus be ignored. For $\vec{p}_{i} \neq \vec{p}_{f}$, the direct terms that may appear correspond, in the framework of the quantum field theory, to unconnected diagrams having no physical sense, and always cancel each other. The cross terms different from zero in the r.h.s. of (8) must verify that the momentum $\vec{p}_{n}$ be equal to $\vec{p}_{f}$ in the first term, and equal to $\vec{p}_{i}$ in the second term. We now insert (8) into (7), and, according to the definition of the states, $E_{n}-E_{f}=\hbar \omega$ for the first term, and $E_{n}-E_{f}=\left(p_{i}^{2}-p_{f}^{2}\right) /(2 m)+\hbar \omega$ for the second one. Let us note that $\left(p_{i}^{2}-p_{f}^{2}\right) /(2 m)=\left(p_{i}-p_{f}\right)\left(p_{i}+p_{f}\right) /(2 m) \sim \hbar k\left(p_{i}+p_{f}\right) /(2 m) \sim \hbar k v_{\mathrm{e}}\left(v_{\mathrm{e}}\right.$ being the electron velocity) which is much smaller than the energy of the photon $\hbar k c$, for nonrelativistic electrons. We can then approximate the denominator of the second term by $\hbar \omega$, and sum in $f, i$ in a trivial manner. Using the expressions (2), eq. (7) finally transforms into

$$
\begin{aligned}
\varphi_{L_{1}}= & -\frac{1}{\hbar} \int_{-\infty}^{+\infty} \mathrm{d} t 2 \operatorname{Re} \frac{e}{m} \sum_{\vec{k}, \vec{\epsilon}} \frac{\hbar}{2 \epsilon_{0} V \omega} \frac{1}{\hbar \omega}\left\langle\psi_{\mathrm{e}}(t)\left|\vec{p} \cdot \vec{\epsilon} e^{i \vec{k} \cdot \vec{r}_{\mathrm{e}}}\right| \psi_{\mathrm{e}}(t)\right\rangle \times \\
& \times \sum_{s} \frac{g \mu_{\mathrm{B}}}{\hbar}\left\langle\chi\left|J_{z}^{(s)} \vec{u}_{z} \cdot\left(-i \vec{k} \times \vec{\epsilon} e^{i \vec{k} \cdot \vec{r}_{s}}\right)\right| \chi\right\rangle,
\end{aligned}
$$

where it is clear that $\varphi_{L_{1}}$ is real. To evaluate the first matrix element of (9), we consider that the wave function of the electron, $\psi_{\mathrm{e}}\left(\vec{r}_{\mathrm{e}}, t\right)$, represents a wave packet, Gaussian for example, centered in $\vec{r}_{0}(t)=\left(x_{0}, y_{0}, 0\right)$, so that $\vec{r}_{\mathrm{e}}=\vec{r}_{0}+\Delta \vec{r}$ and $e^{i \vec{k} \cdot \vec{r}_{\mathrm{e}}}=e^{i \vec{k} \cdot \vec{r}_{0}} e^{i \vec{k} \cdot \Delta \vec{r}}$. Now we must note that the most relevant $\vec{k}$ are those whose wavelengths are of the order of the electron-magnet distance. In consequence, if the electron wave packet is narrow enough so that $|\Delta \vec{r}| \ll d$, where $d$ is the smallest distance (see fig. 1), we can make $e^{i \vec{k} \cdot \Delta \vec{r}} \approx 1$, that is, we can use the electric-dipole approximation. We also use the magnetic-dipole approximation and consider that the elementary (atomic) magnetic-dipole moments are very localized so that the average of the spatial operator $e^{i \vec{k} \cdot \vec{r}_{s}}$ can be approximated by this same expression but with $\vec{r}_{s}$ being the position of the atomic nucleus. Then the operator $H_{\text {int }}^{(\mathrm{m})}$ only acts on spin states. Summing the polarizations, taking into account that $J_{z}^{(s)}|\chi\rangle=\hbar M|\chi\rangle$, and that $(1 / V) \sum_{\vec{k}}$ converts into 
$\left(1 / 8 \pi^{3}\right) \int \mathrm{d}^{3} \vec{k}$, as usual, eq. (9) becomes

$$
\varphi_{L_{1}}=-\frac{1}{\hbar} \int_{-\infty}^{+\infty} \mathrm{d} t 2 \operatorname{Re}\left(-i \frac{e g \mu_{\mathrm{B}} M}{m 16 \pi^{3} \epsilon_{0} c^{2}} \int_{\vec{k}} \frac{\mathrm{d}^{3} \vec{k}}{k^{2}} \vec{p}_{0} \cdot \vec{u}_{z} \times \vec{k} \sum_{s} e^{i \vec{k} \cdot\left(\vec{r}_{0}-\vec{r}_{s}\right)}\right),
$$

where $\vec{p}_{0}=\left\langle\psi_{\mathrm{e}}|\vec{p}| \psi_{\mathrm{e}}\right\rangle$ is the mean value of the momentum of the electron, $\vec{p}_{0}=p_{0} \vec{u}_{y}$. Hence, $\vec{p}_{0} \cdot \vec{u}_{z} \times \vec{k}=p_{0} k_{x}$. If we insert this expression in (10), note that $\vec{r}_{0}-\vec{r}_{s} \equiv\left(x_{0}-x_{s}, y_{0}-y_{s},-z_{s}\right)$, and convert $\sum_{s}$ into the integral $N \int_{\vec{r}} \mathrm{~d}^{3} r$ extended to the volume of the magnet, we obtain

$$
\varphi_{L_{1}}=\frac{1}{\hbar} \int_{-\infty}^{+\infty} \mathrm{d} t \delta E(t), \quad \delta E(t) \equiv \frac{e p_{0} B_{\mathrm{m}}}{8 \pi^{3} m} \operatorname{Im} \int_{\vec{r}} \mathrm{~d}^{3} r \int_{\vec{k}} \mathrm{~d}^{3} k \frac{k_{x} e^{i \vec{k} \cdot \vec{R}}}{k^{2}}
$$

where $B_{\mathrm{m}}=\left(N g \mu_{\mathrm{B}} M / \epsilon_{0} c^{2}\right)$ is the value of the magnetic field inside the magnet, pointing in the $Z$-direction, and $\vec{R} \equiv\left(x_{0}-x, y_{0}-y,-z\right)$. The integral in $\vec{k}$ is straightforward noting that it is $-i\left(\mathrm{~d} / \mathrm{d} x_{0}\right) \int \mathrm{d}^{3} k\left(e^{i \vec{k} \cdot \vec{R}} / k^{2}\right)$ and using polar coordinates with polar axis in the direction of $\vec{R}$. We obtain

$$
\delta E(t)=\epsilon_{0} c^{2} B_{\mathrm{m}} \int_{S} \mathrm{~d} x \mathrm{~d} y \int_{-\infty}^{+\infty} \mathrm{d} z B_{\mathrm{e} z}(\vec{r}, t)=\frac{\epsilon_{0} c^{2}}{2} \int_{\vec{r}} 2 \vec{B}_{\mathrm{m}} \cdot \vec{B}_{\mathrm{e}}(\vec{r}, t) \mathrm{d}^{3} r,
$$

where $S$ is the circular section of the magnet, and

$$
B_{\mathrm{e} z}(\vec{r}, t)=-\frac{\epsilon_{0} c^{2}}{4 \pi} \frac{e p_{0}\left(x_{0}-x\right)}{2 m R^{3}}
$$

is the $z$ component of the magnetic field created by the electron in motion. When (12) and (13) are put in (11) and the integrals performed (the integrals are easier if made in the order: $z$, then $t$, then $x, y)$ we get

$$
\varphi_{L_{1}}=-\frac{e \Phi}{2 \hbar}
$$

where $\Phi=B_{\mathrm{m}} S$ webers is the magnetic flux inside the infinitely long magnet. It is obvious that the change in phase for the path $L_{2}, \varphi_{L_{2}}$, is just eq. (14) with opposite sign since in eq. (13), $x_{0}-x$ changes the sign (the $Y$-axis is now parallel to the direction of path $L_{2}$, and $\left.x_{0}-x<0\right)$. Hence we obtain

$$
\varphi_{L_{2}}-\varphi_{L_{1}}=\frac{e}{\hbar} \Phi,
$$

which is the same result obtained in the semiclassical theory.

Discussion. - The main shortcoming of our calculation is that it is valid only if $\varphi \ll 1$. The validity may be easily extended if we divide the time interval $[-T, T]$ in small intervals by the intermediate times $t_{1}, t_{2}, \ldots t_{n}$. In every small interval, say between $t_{j-1}$ and $t_{j}$, we may apply perturbation theory and arrive at an expression like eq. (10), but with the time integral performed over the small interval, to get the partial change of phase, $\varphi_{j}$. The result is correct if the partial time intervals are not too small, so that the passage from (5) to (7) is still valid. Then, the total change of phase, $\varphi_{L_{1}}$, may be obtained from the equality

$$
\left(1+i \varphi_{1}\right)\left(1+i \varphi_{2}\right) \ldots\left(1+i \varphi_{n}\right) \approx e^{i \sum_{j} \varphi_{j}}=e^{i \varphi_{L_{1}}} .
$$

It is not difficult to see that the procedure leading to eq. (16) takes into account the exchange of any number of photons, in contrast with the simple procedure of the preceding section, which considers single photon exchange. 
This method is not yet exact because it takes into account only cases where every photon is absorbed before the next photon is emitted. Nevertheless we may assume that it is a good enough approximation because the energy denominator (see (9)) is greater if more than one photon is present. After that, we arrive exactly at eq. (10), but now with a validity not restricted to small phase changes.

Our calculation presents a number of interesting features. The interaction, being due to photon exchange, propagates with the velocity of light, and the phase acquired by the electron may be interpreted as the time integral of an interaction energy, $\delta E(t)$, divided by $\hbar$, as is clearly seen in (11). The interaction energy given by the last member of (12) as the source of the AB effect was suggested by Liebowitz [5] and further discussed by Boyer [6]. But these authors associated that energy with the existence of a force on the electron causing a lag effect. From our calculation it may bee seen that the velocity of the center of the wave packet of the electron does not change in time. In fact, in the Heisenberg picture we have $(\mathrm{d} / \mathrm{d} t)\left\langle\Psi\left|\vec{v}_{\mathrm{e}}\right| \Psi\right\rangle=\left\langle\Psi(-T)\left|\mathrm{d} \vec{v}_{\mathrm{e}} / \mathrm{d} t\right| \Psi(-T)\right\rangle=(i / \hbar)\left\langle\Psi(-T)\left|\left[H, \vec{v}_{\mathrm{e}}\right]\right| \Psi(-T)\right\rangle$, where $H$ is the total Hamiltonian (1) and $\vec{v}_{\mathrm{e}}=\left(\vec{p}+|e| \vec{A}\left(\vec{r}_{\mathrm{e}}\right)\right) / m$ is the operator velocity. It is easy to check that only the term $H_{\text {int }}$ does not commute with $\vec{v}_{\mathrm{e}}$, that commutator being a linear combination of creation and annihilation operators of photons. Since the (time-independent) state of the system in the Heisenberg picture is the vacuum radiation state, the average value of the said commutator in that state is zero. Therefore the center of the electron wave packet moves with constant velocity, no force acts on the electron, and there is no time lag, contrary to the Liebowitz-Boyer conjecture.

The microscopic theory of the AB effect may allow refinements which are not so simple in the standard, semiclassical, treatment. For instance we may go beyond the electric-dipole approximation explained after eq. (9), and get corrections due to the form and size of the electron wave packet. Also it is straightforward to take into account the electron spin by just adding the corresponding term to the interaction Hamiltonian (2). This will be interesting for the prediction of the $\mathrm{AB}$ effect with polarized electrons. We may also calculate the possible loss of visibility of the interference pattern due to the spin flip caused in the electron by the interaction with the magnet.

The perturbation method used here for the interaction mediated by virtual photons may be extended to other problems. For instance, we may calculate the change of the wave function of an atom (or molecule) passing near a macroscopic piece of matter. As the atom has a complex internal structure, the interaction may lead to excited states of it, or of the macroscopic body or both, at the expense of the kinetic energy. If the calculation confirms that the effect is not negligible, it will be relevant in experiments of atomic or molecular interferometry.

We acknowledge financial support of DGICYT Project No. PB-95-0594 (Spain). We are very grateful to T. W. MARshALL and J. L. SÁNCHEZ-GómEz for useful comments.

\section{REFERENCES}

[1] Peshinin M. and Tonomura A., The Aharonov-Bohm Effect (Springer-Verlag, Berlin) 1989.

[2] Ehrenberg W. and Siday R., Proc. Phys. Soc. London Ser. B, 62 (1949) 8.

[3] Aharanov Y. and Bohm, Phys. Rev., 115 (1959) 485.

[4] Bocchieri P. and Loinger A., Lett. Nuovo Cimento, 39 (1984) 148.

[5] Liebowitz B., Nuovo Cimento, 38 (1965) 932.

[6] Boyer T. H., Phys. Rev. D, 8 (1973) 1667, 1679; Nuovo Cimento B, 100 (1987) 685.

[7] Mollenstedtand G. and Bayh W., Phys. Blatter, 18 (1962) 299; Naturwiss., 4 (1962) 81. 